\title{
Automatic Parameter Tuning with Metaheuristics of the AODV Routing Protocol for Vehicular Ad-Hoc Networks
}

\author{
José García-Nieto and Enrique Alba \\ Dept. de Lenguajes y Ciencias de la Computación, University of Málaga, \\ ETSI Informática, Campus de Teatinos, Málaga - 29071, Spain \\ \{jnieto, eat\}@lcc.uma.es
}

\begin{abstract}
Communication protocol tuning can yield significant gains in energy efficiency, resource requirements, and the overall network performance, all of which is of particular importance in vehicular ad-hoc networks (VANETs). In this kind of networks, the lack of a predefined infrastructure as well as the high level of dynamism usually provoke problems such as the congestion of intermediate nodes, the appearance of jitters, and the disconnection of links. Therefore, it is crucial to make an optimal configuration of the routing protocols previously to the network deployment. In this work, we address the optimal automatic parameter tuning of a well-known routing protocol: Ad Hoc On Demand Distance Vector (AODV). For this task, we have used and compared five optimization techniques: PSO, DE, GA, ES, and SA. For our tests, a urban VANET scenario has been defined by following realistic mobility and data flow models. The experiments reveal that the produced configurations of AODV significantly improve their performance over using default parameters, as well as compared against other well-known routing protocols. Additionally, we found that PSO outperforms all the compared algorithms in efficiency and accuracy.
\end{abstract}

Keywords: Vehicular Ad Hoc Networks, On Demand Distance Vector Routing Protocol, Metaheuristics, ns-2 Simulator.

\section{Introduction}

Vehicular Ad Hoc Networks (VANETs) [1] are dynamic networks composed of a set of communicating vehicles (nodes) equipped with devices which are able to spontaneously interconnect each other without any pre-existing infrastructure. This means that no service provider is present as it is usual in traditional cellular telephony. The most popular wireless networking technology available nowadays for establishing VANETs is the IEEE 802.11-b WLAN, also known as WiFi (Wireless Fidelity). New standards such as the IEEE 802.11p and WiFi Direct are promising but still not available to perform real tests with them. This implies that vehicles communicate within a limited range while moving, thus exhibiting a topology that may change quickly and in unpredictable ways. 
In such kind of networks, and given of limitations in coverage and energy consumption, optimizing the routing load is a high priority in the protocol design (done offline, previous to its network deployment). As a matter of fact, an optimal configuration can decisively improve QoS indicators such as the packet delivery ratio, the end-to-end delay and, the routing load, with their implications on enlarging bandwidth and lowering the energy consumption. However, the efficient protocol configuration for VANETs without using automatic intelligent design tools is practically impossible because of the enormous number of possibilities. This motivates the use of metaheuristic techniques [2] which appear as well-suited tools to solve this kind of problems. Unfortunately, few related approaches can be found in the specialized literature. Vanhatupa et al. 3 , proposed a flexible Genetic Algorithm for optimizing channel assignment in Mesh wireless networks. In Alba et al. 4, a specialized Cellular Multi-Objective Genetic Algorithm was used for finding an optimal broadcasting strategy in urban Mobile Ah Hoc Networks (MANETs). Due to its specific design, Ant Colony Optimization (ACO) has been successfully adapted for implementing new routing protocols for MANETs (Di Caro et al. [5) , as well as for resource management (Chiang et al. [6]). More recently, Huang et al. [7] proposed a new routing protocol based on a Particle Swarm Optimizer (PSO) to make scheduling decisions for reducing the packet loss rate in a theoretical VANET scenario.

In the present work, instead of the use of an optimization technique itself as a protocol agent, our main contribution consists in improving the performance of an existing well-known routing protocol by optimally tuning its parameters. This protocol lies in the Ad Hoc On Demand Distance Vector (AODV) [8], whose performance is significantly influenced by the choice of its parameters as stated from its very initial definition in the RFC 3561. For this task, we have used and compared five optimization techniques: Particle Swarm Optimization (PSO) 9, Differential Evolution (DE) [10, Genetic Algorithm (GA) 2], Evolutionary Strategy (ES) [2], and Simulated Annealing (SA) 2]. The popular network simulator $n s-2$ [11] is then used in the evaluation of the solutions (tentative routing parameters) generated by the aforementioned techniques, and providing them with the needed fitness values to guide the search.

We have chosen these algorithms because they constitute a representative subset of metaheuristics, with suitable operators for real parameter optimization, and having varied heterogeneous schemes of population and evolution. For our tests, an instance of a VANET scenario has been defined by following realistic mobility and data flow models from the urban area of Málaga in Spain. The experiments reveal that the produced configurations of AODV significantly improve the default performance of the protocol, as well as the performance of other well-known routing protocols (DSR, DSDV, FSR, TORA, and GPSR) [12.

The remaining of this paper is organized as follows. In the next section, the AODV routing protocol is introduced with its main parameters. Section 3 describes the optimization strategy, and Section 4 presents the VANET scenario evaluated here. Experiments, comparisons, and the result analysis are shown in Section 5. Finally, conclusions and further work are drawn in Section 6. 


\section{AODV Parameter Tuning}

Ad hoc On Demand Distance Vector (AODV) is a routing protocol for ad hoc mobile networks presented in 1999 by C. Perkins and E. Royer [13. It is one of the most studied MANET and VANET routing algorithms, often used as a de facto routing protocol. AODV was designed with the aim of reducing the high number of broadcasting packets and the latency described for its precedent: Destination Sequenced Distance Vector (DSDV) 14] routing protocol. AODV is a reactive on demand algorithm, meaning that, as in Dynamic Source Routing (DSR) [15] protocol, it builds routes among nodes only as desired by source nodes. Nevertheless, AODV uses routing tables in intermediate nodes, what makes the route discovery more efficient than in DSR, specifically in extensive networks with a large number of communicating nodes. In addition, AODV is loop-free, self-starting, and capable of both unicast and multicast routing. All these advantages, along with the fact of having lower complexity of storage than others proactive and reactive protocols (TORA, FSR, GPSR, etc.) [12, led us to choose AODV as the routing protocol to work with.

In spite of all these important advantages, one of the main drawbacks of AODV lies in the variability of its performance, which is significantly influenced by the choice of its control parameters $[8$. Discovering the best values to assign to these parameters and understanding their impact on the network behavior tradeoff is still harder. In addition, tunable parameters are often defined without clear default values and even may be defined over an infinite range. Table@shows a set of the main AODV parameters with their default values as specified in RFC 3561. The range of values each parameter can take has been defined here by following AODV restrictions, with the aim of avoiding pointless configurations.

This way, we can use this set of parameters as a real vector solution to be automatically fine-tuned by an optimization technique, hopefully obtaining a considerably better configuration than the one of default parameters for a given VANET scenario. Additionally, analytic comparisons of both default and optimized AODV configurations as the ones done in this article can help the specialists to identify the main source of problems and assist them in the design of sophisticated routing protocols.

Table 1. Main AODV Parameters. Default values following the RFC 3561 specification.

\begin{tabular}{lll}
\hline Parameter & Default Values & Range \\
\hline ACTIVE_ROUTE_TIMEOUT & $3.0 \mathrm{~s}$ & $1.0 \cdots 10.0$ \\
ALLOWED_HELLO_LOSS & 2 HELLO packets & $1 \cdots 10$ \\
MY_ROUTE_TIMEOUT & $2.0 \times$ ACTIVE_ROUTE_TIMEOUT & $1.0 \cdots 10.0$ \\
NET_DIAMETER & 35 nodes & $1 \cdots 50$ \\
NODE_TRAVERSAL_TIME & $0.04 \mathrm{~s}$ & $0.01 \cdots 1.0$ \\
NET_TRAVERSAL_TIME & $2.0 \times$ NODE_TRAVERSAL_TIME & $1.0 \cdots 10.0$ \\
RREQ_RETRIES & $\times$ NET_DIAMETER & $1 \cdots 10$ \\
RREQ_RATELIMIT & 2 tries & $1.0 \cdots 10.0$ \\
TTL_START & $10.0 \mathrm{kbps}$ & $1.0 \cdots 10.0$ \\
TTL_INCREMENT & $1.0 \mathrm{~s}$ & $1.0 \cdots 10.0$ \\
TTL_THRESHOLD & $2.0 \mathrm{~s}$ & $1.0 \cdots 20.0$ \\
\hline
\end{tabular}


In order to compare different AODV routing configurations (solutions), we have measured the resulted network performance (quality of service) by means of three commonly used metrics in this area [16]:

- Packet delivery ratio (PDR). Fraction of the data packets originated by an application that a routing protocol delivers. A data packet is counted as delivered when it is received complete and correct by the destination node.

- Normalized routing load (NRL). Ratio of administrative routing packet transmissions to data packets delivered. When counting transmissions, each hop is counted separately.

- Average End-to-End delay of a data packet (AEED). Average difference between the time the first data packet is originated by an application and the time this packet is received at its destination.

\section{Optimization Strategy}

Our optimization strategy is composed by basically two main parts: an optimization algorithm and a simulation procedure. The optimization part is carried out by (independently) one of the selected metaheuristic methods. All of them are specially adapted to find optimal (or quasi-optimal) solutions in continuous search spaces (which is the case in this work). The simulation procedure is the way of assigning a quantitative quality value (fitness) to the factors regulating AODV, thus leading to optimal configurations of this protocol tailored to a given VANET scenario instance. This procedure is carried out by means of the ns-2 [1] network simulator, which has been modified in order to accept new routing parameters automatically for this present and similar future research.

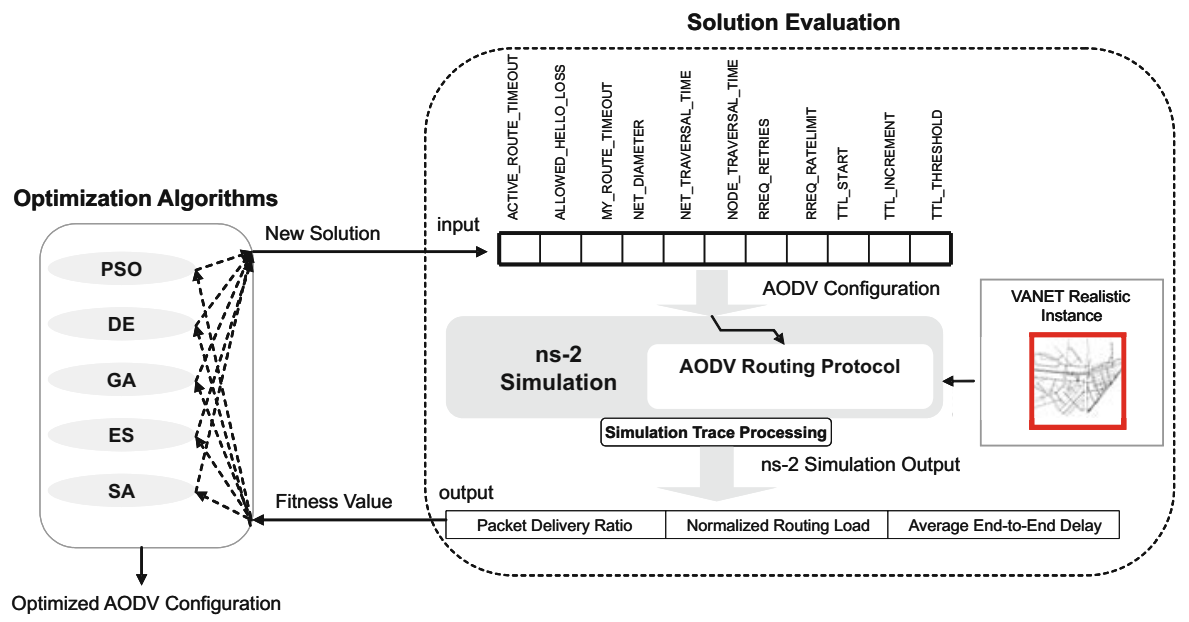

Fig. 1. Optimization strategy for AODV configuration in VANETs. The algorithms invoke the $n s-2$ simulator for each solution evaluation. 
In each optimization algorithm, the evaluation of a solution is carried out by means of the simulation component. As Fig. 1illustrates, when a given algorithm generates a new solution it is immediately used for configuring the AODV. Then, $n s-2$ is started and emulates the VANET scenario instance, taking its time in evaluating the scenario with buildings, signal loss, obstacles, traffic lights, vehicles, speed, covered area, etc., under the circumstances defined by the routing parameters of AODV from the algorithm. After the simulation, ns-2 returns the global information about the Packet Delivery Ratio (PDR), the Normalized Routing Load (NRL), and the Average End-to-End Delay (AEED) of the whole mobile WiFi scenario (simulating 50 independent application sessions for each fitness computation). This information is used to compute the fitness function as follows:

$$
\text { fitness }=w_{1} \cdot(-P D R)+w_{2} \cdot N R L+w_{3} \cdot A E E D \cdot C
$$

The objective here consists in maximizing PDR, and minimizing both, NRL and AEED. As expressed in Equation[1, we used an aggregative minimizing function, and for this reason PDR was formulated with a negative sign. In this equation, factors $w_{1}, w_{2}$, and $w_{3}(0.5,0.3$ and 0.2 , respectively) were used for weighing the influence of each metric on the resulted fitness value. This way, PDR takes priority over NRL and AEED since we firstly look for the routing effectiveness and secondly (but also important) for the communication efficiency. AEED is also multiplied by a constant $C=0.01$ in order to deal with similar range to PDR and NRL.

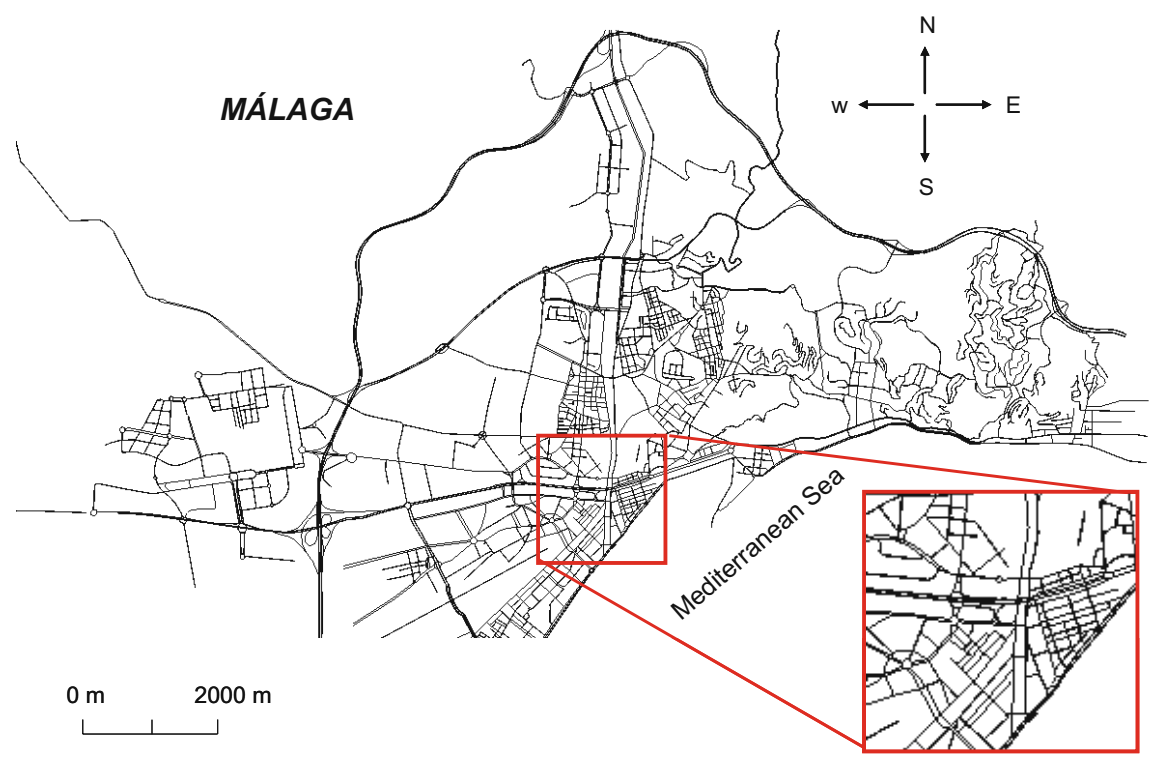

Fig. 2. Málaga real VANET scenario. Selected surface $(2,000 \times 2,000$ meters $)$ in the downtown city. 


\section{VANET Scenario and Mobility Models}

Since real vehicular traces are not available, and the generation of a real VANET scenario requires a great number of resources and people, we can use a traffic/network simulator to perform the movement of vehicles as well as the communication activity between them. Furthermore, we can generate realistic VANET environments by automatically selecting real city areas (taking into account road directions, signal lights, and traffic rules) from digital maps, and finally apply a realistic mobility and communication model to each vehicle agent.

Following this idea, we have generated in this work a VANET instance by mapping a metropolitan area of 2,000×2,000 $\mathrm{m}^{2}$ from the city of Málaga (Spain). For this task, we first used the SUMO car traffic simulator [17] for describing in XML format the step by step movement of each vehicle within a 300 second time period. Fig. 2 shows the complete map of Málaga processed with SUMO traffic simulator (selected area is expanded in this figure). To use that traffic model we exported the XML input from SUMO into an ns-2 [11] simulator movement pattern in Tcl format. A number of 50 vehicles are involved in the simulation with 4050 recorded vehicles direction/speed changes. Through the simulation time, we captured different levels of car density (between 15 and 45 vehicles $/ \mathrm{km}^{2}$ ), car speed (between 10 and $50 \mathrm{~km} / \mathrm{h}$ ), and network activity (from 2 to 50 connections).

The data flow model performs 50 sessions of the CBR (Constant Bit Rate) network application over UDP source agents in vehicles, thus interconnecting to each other by following our mobility model. The CBR data packet size is 512 bytes and packet rate is 4 packets per second. The remaining of simulation parameters are summarized in Table 2 for future reproduction purposes. We have chosen a fixed data rate since we do not aim to study the maximum throughput, but we want to investigate the ability of AODV to successfully find and maintain routes in a given VANET.

Table 2. Simulation parameters in $n s-2$

\begin{tabular}{ll}
\hline Parameter & Value \\
\hline Simulation time & $300 \mathrm{~s}$ \\
Simulation area & $2,000 \times 2,000 \mathrm{~m}^{2}$ \\
Number of vehicles & 50 \\
Vehicle speed & $10-50 \mathrm{~km} / \mathrm{h}$ \\
Propagation model & Two Ray Ground \\
Radio frequency & $2.47 \mathrm{GHz}$ \\
Channel bandwidth & $5 \mathrm{Mbps}$ \\
Mac protocol & $802.11-\mathrm{b}$ \\
Transmission range of vehicles & $250 \mathrm{~m}$ \\
CBR data flow & 50 sessions \\
\hline
\end{tabular}




\section{Experiments}

We have conducted a series of experiments by using the implementation of the five algorithms (PSO, DE, GA, ES, and SA) provided by MALLBA [18], a C++ based framework of metaheuristics for solving optimization problems. The simulation phase was carried out by running $n s-2$ simulator v-2.31. For the experiments, we have made 30 independent runs of each optimization algorithm on machines with a Pentium IV $2.4 \mathrm{GHz}$ core, 1 GB of RAM, and O.S. Linux Fedora core 6. Each one of these independent runs performs different vehicular mobility and communication patters based on independent random seeds inside each (ns-2) simulation, hence contributing to the generalization of the results. Therefore, a total of 30 different communication/mobility scenarios are analyzed for every optimization algorithm (we have 5 algorithms in our study).

\subsection{Parameter Settings of the Optimization Algorithms}

All studied algorithms were configured in order to perform 5,000 solution evaluations per run. Population based algorithms (PSO, DE, GA, and ES) were configured with 20 individuals. Since these algorithms perform quite different operations, we have set the parameters after preliminary executions where the computational effort in terms of time and number of evaluations was balanced. Table 3 summarizes the parameter setting specific to each algorithm.

Table 3. Summarized parameter settings of the optimization algorithms

\begin{tabular}{llllc}
\hline Algorithm & Operator & Parameter & Symbol & Value \\
\hline \multirow{2}{*}{ PSO } & \multirow{2}{*}{ Velocity Update } & Local Coefficient & $\varphi_{1}$ & 2.0 \\
& & Social Coefficient & $\varphi_{2}$ & 2.0 \\
& & Inertia Weigh & $w$ & 0.5 \\
\hline \multirow{2}{*}{ DE } & Differential Crossover & Crossover Probability & $C r$ & 0.9 \\
& Differential Mutation & Mutation Factor & $\mu$ & 0.1 \\
\hline \multirow{2}{*}{ GA } & Uniform Crossover & Crossover Probability & $P_{\text {cros }}$ & 0.8 \\
& Uniform Mutation & Mutation Probability & $P_{m u t}$ & 0.2 \\
\hline \multirow{2}{*}{ ES } & Replacement & Replacement Strategy & $(\mu, \lambda)$ & $(20,20)$ \\
& Correlated Mutation & Mutation Probability & $P_{m u t}$ & 0.1 \\
& Correlated Crossover & Crossover Probability & $P_{\text {cros }}$ & 0.9 \\
\hline \multirow{2}{*}{ SA } & Solution Update & Temperature Decay & $T$ & 0.8 \\
\hline
\end{tabular}

\subsection{Simulation Results and Comparisons}

Table 4 contains the results obtained after the experimentation. The second column contains both, the mean and the standard deviation (std) of the resulted best fitness values (out of 30 independent runs) for each one of the five optimization algorithms. In order to provide statistical meaningful comparisons, we have applied Friedman and Signed Ranked (Wilcoxon) statistical tests [19] to the numerical distributions of results. We have used these non-parametric tests since resulted distributions usually violate the condition of normality required to apply parametric tests $(\mathrm{Z}$ Kolmogorov-Smirnov $=0.003)$. The confidence level was set to $95 \%$ ( $p$-value $=0.05)$, which allows us to ensure that these results are statistically different if they result in $p$-value $<0.05$. 
Table 4. Results obtained by the five optimization algorithms when optimizing AODV on the Málaga VANET instance

\begin{tabular}{l|r|r|c|c}
\hline Alg. & Mean $_{\text {std }}$ & Best & Fried. & Wilcox.p \\
\hline PSO & $\mathbf{- 1 3 . 5 5} \pm \mathbf{1 . 2 4}$ & $\mathbf{- 1 5 . 3 4}$ & $\mathbf{1 . 4 3}$ & - \\
DE & $-12.93 \pm 0.76$ & -14.35 & 1.73 & $2.18 \mathrm{e}-02$ \\
ES & $-10.53 \pm 1.74$ & -12.67 & 3.13 & $1.73 \mathrm{e}-06$ \\
SA & $-6.12 \pm 6.38$ & -12.76 & 4.10 & $2.87 \mathrm{e}-06$ \\
GA & $-5.88 \pm 2.81$ & -10.38 & 4.60 & $1.92 \mathrm{e}-06$ \\
\hline
\end{tabular}

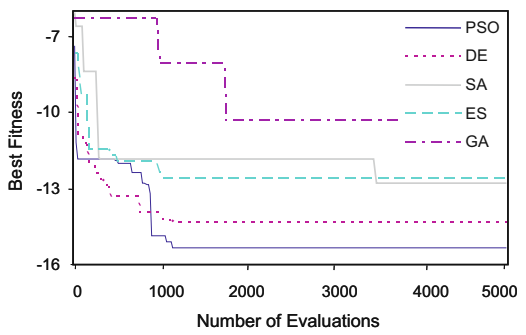

Several observations can be made from these results. First of all, the best mean fitness $(-13.55 \pm 1.24)$ was reached by PSO, which also shows a reasonably low value of standard deviation. In addition, this algorithm has obtained the solution with the overall minimum fitness $(-15.34)$, which corresponds with the AODV configuration that shows the best tradeoff in quality indicators $(P D R=60 \%$, $N R L=43.06$, and $A E E D=866.02)$ for the studied VANET. These results are statistically tested in column 4 where we can check that PSO effectively shows the lowest rank according to Friedman test (the lower, the better).

Second, DE obtained slightly higher mean fitness $(-12.93 \pm 0.76)$ than PSO, but with the lowest value of standard deviation. In spite of its moderate performance (ranked as second in Friedman test), DE shows the most robust behavior for this instance. The worst rank was obtained by GA, although showing in this case a lower standard deviation than SA. In this sense, we suspect that the trajectory search mechanism of SA can deteriorate the robustness for this problem. The graphic plotted below shows the trace of the Best performed runs of each algorithm where we can easily observe the early convergence and better behavior of PSO and DE with regard to SA, ES, and GA.

A last observation concerns the Signed Rank test (Wilcox.p in Table 4), for which we have used PSO as control algorithm (the one with best rank) in order to confirm whether differences in distribution of results can be found or not. As we can observe in column 5, all algorithms obtained statistically worse results than PSO since $p$-values of distributions refuse the null hypothesis $(<0.05)$. Then we can state that, for the studied VANET instance, PSO shows the best performance compared to the rest of algorithms in the configuration of the AODV protocol.

\subsection{QoS Analysis}

After the analysis of the algorithms themselves, in this section we compare the results in terms of quality of service indicators. This comparison constitutes the main contribution of this work. Therefore, Table 5 shows the results of applying a set of well-known routing protocols to our VANET instance (Málaga), including AODV with its default configuration (RFC 3561). These protocols are: Dynamic Source Routing (DSR), Destination Sequence Distance Vector (DSDV), Fisheye State Routing (FSR), Temporally Ordered Routing (TORA), and Greedy Perimeter Stateless (GPSR). The best AODV configurations obtained by all studied algorithms are deployed in the bottom second half of Table 5 . 
Table 5. Comparison with other routing protocols

\begin{tabular}{l|r|rrr}
\hline Protocol & fitness & $P D R$ & $N R L$ & $A E E D$ \\
\hline AODV & -5.32 & $60.00 \%$ & $75.33 \mathrm{kbps}$ & $1,038.79 \mathrm{~ms}$ \\
DSR & -5.10 & $42.00 \%$ & $45.95 \mathrm{kbps}$ & $1,055.68 \mathrm{~ms}$ \\
DSDV & -4.17 & $28.00 \%$ & $0.00 \mathrm{kbps}$ & $4,913.43 \mathrm{~ms}$ \\
FSR & +0.53 & $20.00 \%$ & $0.00 \mathrm{kbps}$ & $5,268.15 \mathrm{~ms}$ \\
TORA & +6.57 & $66.66 \%$ & $133.00 \mathrm{kbps}$ & $4.48 \mathrm{~ms}$ \\
GPSR & +47.03 & $100.00 \%$ & $332.00 \mathrm{kbps}$ & $143.27 \mathrm{~ms}$ \\
\hline AODV $_{P S O}$ & $-\mathbf{1 5 . 3 4}$ & $60.00 \%$ & $43.06 \mathrm{kbps}$ & $866.02 \mathrm{~ms}$ \\
AODV $_{D E}$ & -14.35 & $62.00 \%$ & $47.06 \mathrm{kbps}$ & $1,271.45 \mathrm{~ms}$ \\
AODV $_{S A}$ & -12.76 & $60.00 \%$ & $47.00 \mathrm{kbps}$ & $1,552.67 \mathrm{~ms}$ \\
AODV $_{E S}$ & -12.67 & $64.00 \%$ & $53.43 \mathrm{kbps}$ & $1,644.97 \mathrm{~ms}$ \\
AODV $_{G A}$ & -10.38 & $62.00 \%$ & $59.32 \mathrm{kbps}$ & $1,409.79 \mathrm{~ms}$ \\
\hline
\end{tabular}

As we can clearly observe, all the AODV configurations computed by the metaheuristics obtained better fitness when compared to competitor routing protocols, including AODV with default parameters. This is a true improvement since even GA, the algorithm with the worst performance here, can generate a set of parameters that helps AODV to outperform all compared protocols. As to the best configuration, AODV $P S O$, the protocol performance was improved by decreasing both, the NRL by $42.83 \%$ (from $75.33 \mathrm{kbps}$ to $43.06 \mathrm{kbps}$ ) and the AEED by $16.63 \%$ (from 1,038.79 ms to $866.02 \mathrm{~ms}$ ), whilst showing the same PDR as default AODV. Concerning other protocols, GPSR and TORA show a high packet delivery $(100 \%)$ but provoking the overhead of the network (NRL $=332.00$ kbps). On the contrary, DSDV and FSR keep reduced the network overload but at the cost of performing a low ratio of packet delivery (PDR $\leq 28 \%)$. All this contrasts with our AODV $P S O$, which always obtains percentages of PDR higher than $60 \%$ plus optimizing both, the network load and end-to-end delay, a capital need for an efficient performance in large VANETs scenarios. In summary, all these observations give us a real insight on the advantage of using PSO (and metaheuristics in general) for the tuning of routing protocols.

\section{Conclusions}

In this work we have addressed the optimal parameter tuning of the routing protocol AODV. For this task, we have used and compared five optimization techniques: PSO, DE, GA, ES, and SA. An instance of VANET for urban scenario has been defined by following realistic mobility and data flow models. The experiments reveal that the produced configurations of AODV significantly improve its performance with respect to using default parameters, as well as the performance of other well-known routing protocols. Specifically, for AODV, the routing load and the end-to-end delay were decreased by $42.83 \%$ and $16.63 \%$, respectively. In addition, we found that PSO outperforms all the compared algorithms. As a future work we are presently studying the use of Multiobjective metaheuristics in the optimal configuration of routing protocols. 
Acknowledgments. Authors acknowledge funds from the CICE, Junta Andalucia, under contract P07-TIC-03044 (DIRICOM project http://diricom.lcc. uma.es) and Spanish Ministry of Sciences and Innovation (MICINN) and FEDER under contract TIN2008-06491-C04-01 (M* project http://mstar.lcc.uma.es). José García-Nieto is supported by grant BES-2009-018767 from the MICINN.

\section{References}

1. Härri, J., Filali, F., Bonnet, C.: Mobility Models for Vehicular Ad Hoc Networks: A Survey and Taxonomy. Research Report RR-06-168 (March 2007)

2. Blum, C., Roli, A.: Metaheuristics in combinatorial optimization: Overview and conceptual comparison. ACM Computing Surveys 35(3), 268-308 (2003)

3. Vanhatupa, T., Hännikäinen, M., Hämäläinen, T.: Optimization of mesh WLAN channel assignment with a configurable genetic algorithm. In: WiMeshNets 2006 (2006)

4. Alba, E., et al.: A Cellular MOGA for Optimal Broadcasting Strategy in Metropolitan MANETs. Computer Communications 30(4), 685-697 (2007)

5. Di Caro, G.A., Ducatelle, F., Gambardella, L.M.: AntHocNet: An Adaptive NatureInspired Algorithm for Routing in Mobile Ad Hoc Networks. European Transactions on Telecommunications 16(5), 443-455 (2005)

6. Chiang, F., Chaczko, Z., Agbinya, J., Braun, R.: Ant-based topology convergence algorithms for resource management in VANETs. In: Moreno Díaz, R., Pichler, F., Quesada Arencibia, A. (eds.) EUROCAST 2007. LNCS, vol. 4739, pp. 992-1000. Springer, Heidelberg (2007)

7. Huang, C., Chuang, Y., Hu, K.: Using particle swarm optimization for QoS in ad-hoc multicast. Eng. Appl. of Artificial Intelligence (2009) (in Press)

8. Perkins, C.E., Belding-Royer, E.M., Das, S.: Ad Hoc on Demand Distance Vector (AODV) Routing. IETF RFC 3561 (2003), http://moment.cs.ucsb.edu/pub/rfc3561.txt

9. Kennedy, J., Eberhart, R.: Particle Swarm Optimization. In: IEEE International Conference on Neural Networks, November 1995, vol. 4, pp. 1942-1948 (1995)

10. Price, K.V., Storn, R., Lampinen, J.: Differential Evolution: A practical Approach to Global Optimization. Springer, London (2005)

11. The Network Simulator Project - Ns-2, http://www.isi.edu/nsnam/ns/

12. Toh, C.: Ad Hoc Wireless Networks: Protocols and Systems. Prentice Hall PTR, Upper Saddle River (2001)

13. Perkins, C.E., Royer, E.M.: Adhoc On Demand Distance Vector Routing. In: 2nd IEEE Workshop on MCSA, Metz, France, pp. 90-100 (1999)

14. Perkins, C.E., Bhagwat, P.: Highly Dynamic Destination-Sequenced DistanceVector Routing (DSDV) for Mobile Computers. In: ACM SIGCOMM 1994, London, UK, pp. 234-244 (1994)

15. Johnson, D.B., Maltz, D.A., Broch, J.: DSR: the dynamic source routing protocol for multihop wireless ad hoc networks. In: Ad hoc Networking. Addison-Wesley Longman Publishing Co., Inc., Boston (2001)

16. Naumov, V., Baumann, R., Gross, T.: An evaluation of inter-vehicle ad hoc networks based on realistic vehicular traces. In: Proceedings of the 7th ACM MobiHoc, pp. 108-119. ACM, New York (2006)

17. Krajzewicz, D., Bonert, M., Wagner, P.: The open source traffic simulation package SUMO. In: RoboCup 2006, Bremen, Germany, pp. 1-10 (2006)

18. Alba, E., Luque, G., García-Nieto, J., Ordonez, G., Leguizamón, G.: MALLBA: A software library to design efficient optimisation algorithms. Int. Journal of Innovative Computing and Applications (IJICA) 1(1), 74-85 (2007)

19. Wilcox, R.: New statistical procedures for the social sciences, Hillsdale (1987) 